\title{
Endometriosis intestinal, una causa de suboclusión intestinal poco sospechada. Serie de casos
}

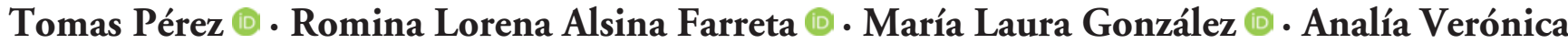

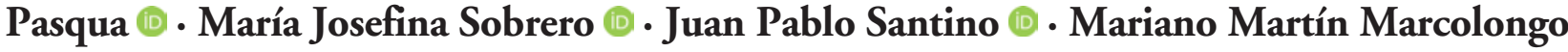

Hospital Italiano de Buenos Aires. Ciudad Autónoma de Buenos Aires, Argentina.

Acta Gastroenterol Latinoam 2021;51(4):431-436

Recibido: 21/04/2021 / Aceptado: 05/11/2021 / Publicado online: 13/12/2021 / https://doi.org/10.52787/RJPF3089

\section{Resumen}

La endometriosis se define como la presencia de glándulas endometriales en sitios extrauterinos. Se considera endometriosis infiltrante profunda intestinal cuando la lesión endometriósica se encuentra situada a más de $5 \mathrm{~mm}$ por debajo del peritoneo. La obstrucción intestinal por endometriosis es poco frecuente dado que ocurre aproximadamente en el 1\% de los casos de endometriosis infiltrante profunda intestinal. Presentamos los casos de tres pacientes con oclusión intestinal como debut de esta entidad. Su presentación sintomática puede mimetizar múltiples entidades de la gastroenterología, principalmente el sindrome de intestino irritable o la enfermedad inflamatoria intestinal, por lo que su diagnóstico puede resultar dificultoso. Sospechar esta patología resulta importante para definir la conducta terapéutica y mejorar la calidad de vida de nuestros pacientes.

Palabras claves. Endometriosis, suboclusión intestinal, dolor abdominal, intestino irritable.

Correspondencia: Pérez Tomas

Correo electrónico: tomas.perez@hospitalitaliano.org.ar

\section{Intestinal Endometriosis, an Under- Suspected Cause of Intestinal Subocclusion: A Case Series}

\section{Summary}

Endometriosis is defined as the presence of endometrial glands in extrauterine sites. Intestinal deep infiltrating endometriosis is considered when the endometriotic lesion is located more than $5 \mathrm{~mm}$ below the peritoneum. Intestinal obstruction due to endometriosis is rare, occurring in approximately $1 \%$ of cases. We present three cases with intestinal occlusion as deep infiltrating endometriosis debut. Its symptomatic presentation can mimic multiple gastroenterological entities, mainly irritable bowel syndrome or inflammatory bowel disease so its diagnosis can be difficult. However, suspecting this pathology and diagnosing it is important to define therapeutic behavior and improve the quality of life of our patients.

Keywords. Endometriosis, Intestinal Suboclusión, Abdominal Pain, Irritable Bowel Syndrome.

\footnotetext{
Abreviaturas

EIPI: Endometriosis de infiltración profunda intestinal.

SII: Sindrome de intestino irritable.

EII: Enfermedad inflamatoria intestinal.

DIE: Deep infiltrating intestinal endometriosis.

RNM: Resonancia nuclear magnética.

TVUS: Ecografia transvaginal.
} 


\section{Introducción}

$\mathrm{La}$ endometriosis es una enfermedad benigna caracterizada por la confirmación histológica de glándulas endometriales y/o estroma endometrial en sitios extrauterinos. ${ }^{1,2}$

Esta entidad afecta al $10 \%$ de las mujeres en edad reproductiva y es causa de dolor abdominal pelviano crónico e infertilidad. ${ }^{3,4}$

La endometriosis infiltrante profunda intestinal (EIPI) se define como la lesión que infiltra, al menos, la capa muscular de la pared intestinal. La mucosa está infiltrada en menos del 5\% de las lesiones intestinales. Fuera de la pared intestinal, la endometriosis profunda se define como una masa endometriósica sólida situada a más de $5 \mathrm{~mm}$ de profundidad en el peritoneo.,

La afectación intestinal ocurre en el $12 \%$ de los pacientes y es el sitio extragenital más frecuente. Las localizaciones anatómicas predominantes son en primer lugar el recto, seguido del colon sigmoides, el apéndice y, en último lugar, el intestino delgado. ${ }^{1-6}$

Esta entidad debe sospecharse en mujeres con dismenorrea, dispareunia y dolor pelviano crónico, pudiendo ser diagnóstico diferencial de cuadros como el síndrome de intestino irritable (SII) o la enfermedad inflamatoria intestinal (EII). En ocasiones se presenta con hematoquezia o como cuadros suboclusivos caracterizados por náuseas y vómitos. ${ }^{7}$

La oclusión intestinal es más frecuente cuando se asocia la infiltración de la pared intestinal con una angulación fija, como sucede en el compromiso del íleon terminal y de la válvula ileocecal. ${ }^{8}$

La resonancia nuclear magnética (RNM) con gel vaginal presenta una sensibilidad y especificidad cercanas al $90 \%$, por lo que es una de las técnicas más utilizadas para el diagnóstico de esta entidad, pudiendo identificarse masas o focos hiperintensos en T1 que sugieren fuertemente la presencia de focos hemorrágicos secundarios a endometriosis. ${ }^{9-12}$

La ecografía transvaginal (TVUS) tiene una alta precisión para identificar la presencia de endometriosis pélvica y estimar la profundidad de invasión de la pared intestinal, con una sensibilidad del $83 \%$ y una especificidad del $94 \% .^{9-12}$

La complejidad de esta enfermedad está determinada por la variedad de presentaciones clínicas: su multifocalidad y multicentricidad, la participación de sitios no ginecológicos y la dificultad en el diagnóstico. ${ }^{9-12}$

Presentamos el caso de tres mujeres con oclusión intestinal como debut de EIPI en nuestra institución, una entidad infrecuente y poco sospechada, que constituye uno de los diagnósticos diferenciales al analizar patologías intestinales.

\section{Caso $N^{\circ} 1$}

Paciente de sexo femenino de 34 años de edad, con antecedente de colon irritable desde los 18 años, que consulta por dolor abdominal, náuseas y diarrea intermitente. Estos cuadros ocurrían de forma esporádica y no presentaban correlación temporal con el ciclo menstrual. Presenta un laboratorio sin hallazgos de relevancia, incluyendo anticuerpos antitransglutaminasa y anti DGP negativos. Se realiza una videoendoscopía digestiva alta (Figura 1), con biopsias de la $2^{\text {da }}$ porción duodenal, sin atrofia vellositaria, y una videocolonoscopía completa, sin particularidades (Figura 2).

Figura 1. Videoendoscopia digestiva alta: mucosa de aspecto normal.

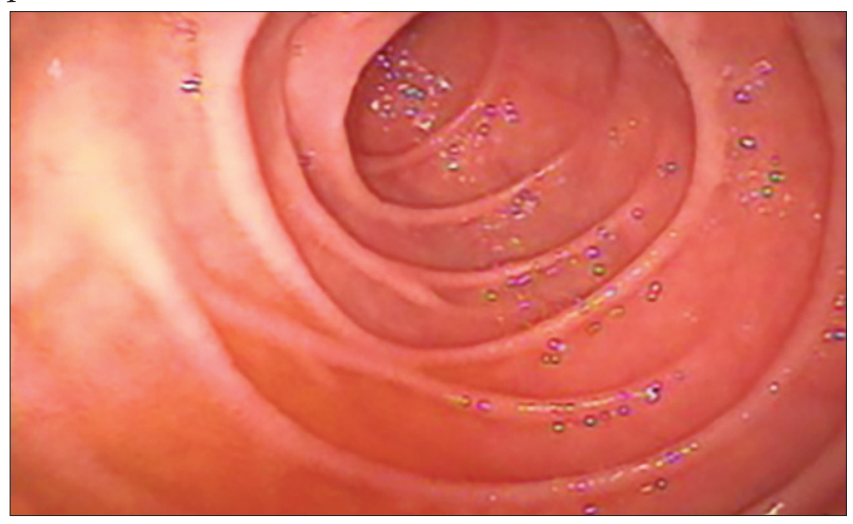

Figura 2. Videocolonoscopía. Válvula ileocecal: mucosa de aspecto normal.

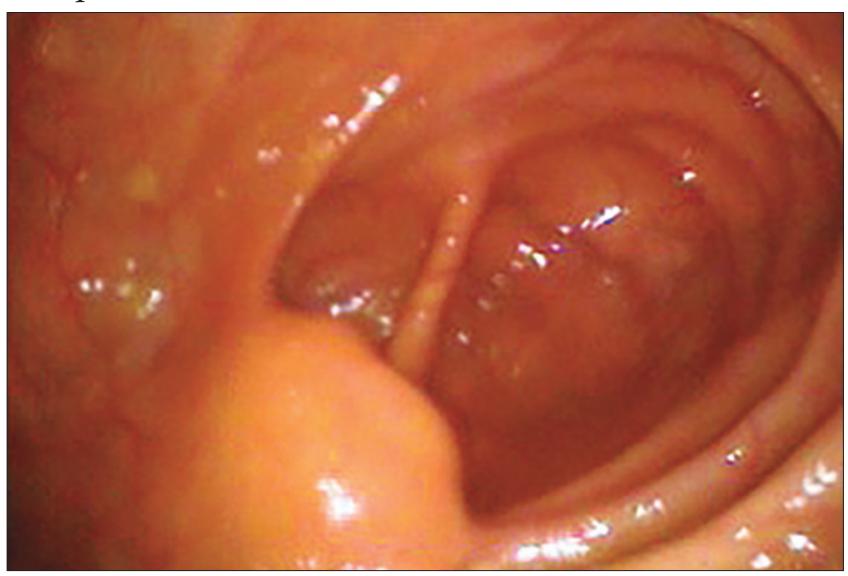

A los diez días consulta nuevamente por cuadro de suboclusión intestinal, afebril, sin diarrea ni signos de sangrado digestivo. Se realiza un laboratorio en el que no se evidencia anemia ni leucocitosis, y presenta además parámetros inflamatorios dentro de los valores normales: ESD $12 \mathrm{~mm}$ (VN: 2-20) y PCR US 1.2 (VN: 1- 5 mg/L). En este contexto, se le realiza una tomografía de abdomen con contraste, en la que se observa una distensión de las asas del intestino delgado a expensas de una lesión estenosante simétrica que compromete el íleon distal próximo a la válvula ileocecal (Figura 3). 
Se decide realizar una laparoscopía, en la que se visualizan las asas del intestino delgado distendidas a partir de la válvula ileocecal, con punto claro de transición a ese nivel, asociado con una lesión duro-pétrea, que compromete el íleon terminal, y múltiples formaciones ganglionares a nivel de la cadena ileocólica. Se realiza una hemicolectomía derecha (Figura 4).

Figura 3. Tomografía computada. Distensión de las asas del intestino delgado por lesión estenosante, que compromete el ileon distal próximo a la válvula ileocecal (flecha).

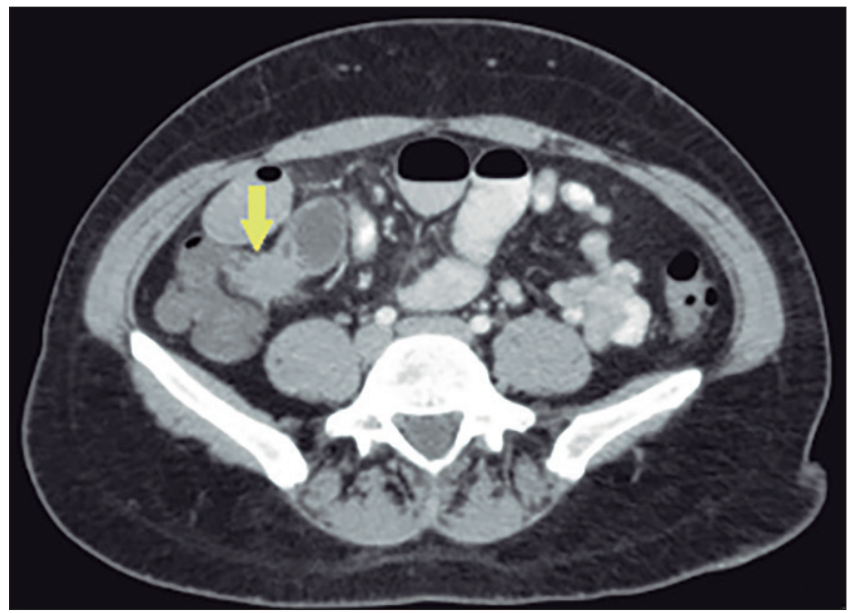

Figura 4. Pieza quirúrgica. Lesión dura pétrea que compromete ileon terminal. Al corte, lesión ulcerada que ocupa el 20\% de la circunferencia.

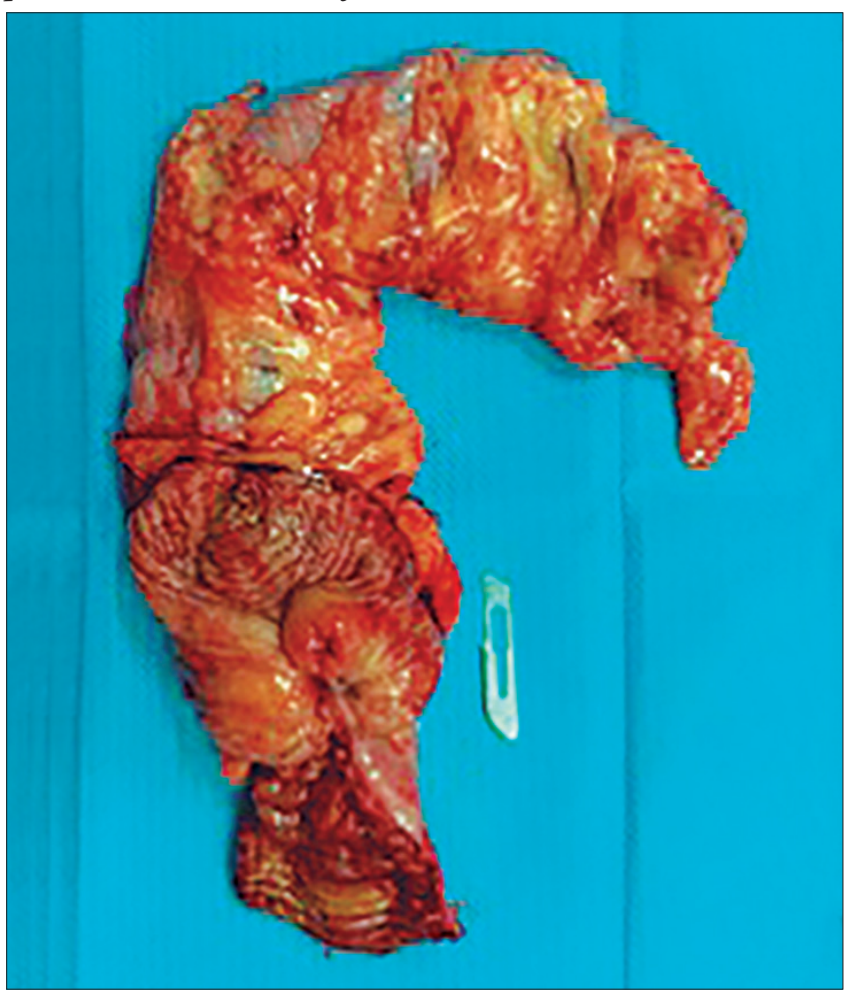

La paciente evoluciona en el posquirúrgico inmediato de manera favorable, obteniéndose la anatomía patológica que informa endometriosis ileocólica, con coexistencia de focos glandulares y apendiculares (Figura 5).

Se realiza ecografía transvaginal de manera ambulatoria, en la que no se evidencian nuevos focos endometriósicos, y se encuentra en plan de realizar una resonancia nuclear magnética para descartar focos aún más profundos.

Actualmente se encuentra en seguimiento por el servicio de Ginecología, realizando tratamiento con Dienogest/Etinilestradiol, con una rotunda mejoría de la sintomatología.

Figura 5. Hematoxilina y eosina. 100x. Se observa mucosa colónica normal en la porción superior. En el espesor de la muscular de la mucosa, en la parte inferior derecha de la imagen, se observan glándulas endometriales (flecha).

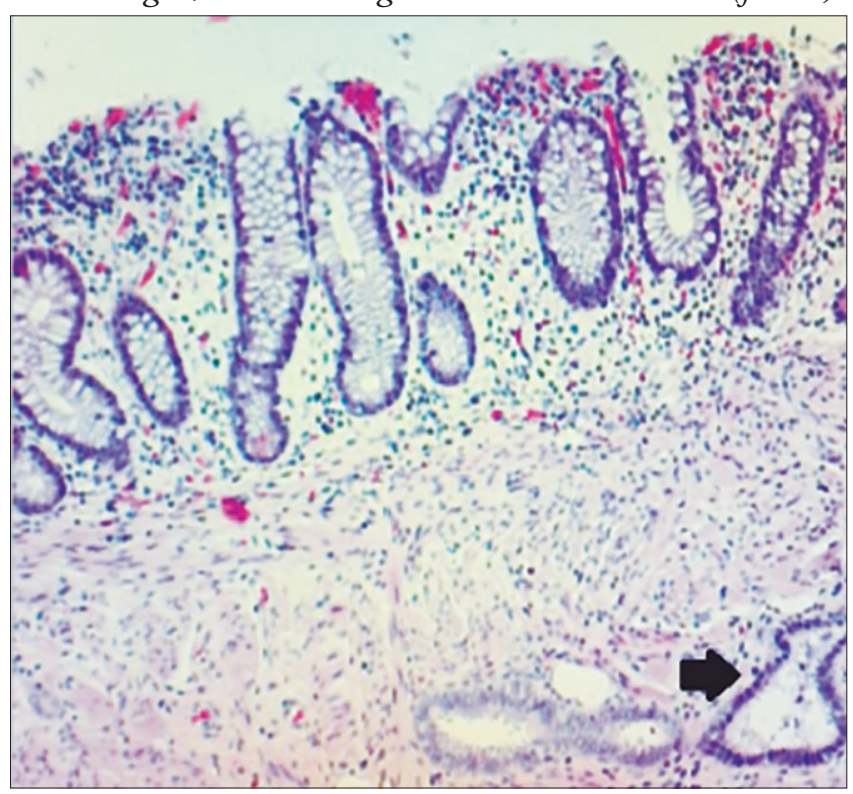

\section{Caso $\mathbf{N}^{\circ} 2$}

Paciente de sexo femenino de 34 años de edad, con historia de dolor abdominal de tipo cólico asociado a distensión, diarrea y vómitos que se presentan de forma esporádica e intermitente, con resolución espontánea. Esta sintomatología la presentó durante los últimos seis años $y$, en general, se encontraba asociada a episodios de estrés como principal desencadenante. La paciente niega relación de los síntomas con la ingesta, el ciclo menstrual u otros eventos.

En forma ambulatoria se solicitan estudios para descartar patología orgánica. Se realiza coprocultivo, que resulta negativo, toxina de Clostridium difficile en materia fecal no reactiva, leucocitos en materia fecal ausentes, perfil de enfermedad celíaca sin particularidades, PCR US 
1.90 (VN: 1- $5 \mathrm{mg} / \mathrm{L})$. Además, se solicita una ecografía abdominal, que revela engrosamiento de las asas del intestino delgado y grueso, y estudios endoscópicos que solamente evidencian gastritis como hallazgo patológico.

En este contexto, se solicita una resonancia de abdomen y pelvis, que se suspende por intolerancia y negativa de la paciente para continuar con los estudios diagnósticos.

Luego de un mes, la paciente concurre a la guardia por intensificación del dolor abdominal, asociado a intolerancia digestiva, vómitos alimentarios, marcada distensión abdominal y deposiciones escasas de consistencia líquida de varias semanas de evolución, asociado a pérdida de $5 \mathrm{~kg}$ de peso. Se realiza una tomografía de abdomen en la que se observa una marcada dilatación de las asas del intestino delgado, con contenido líquido y transición asa fina - asa dilatada a nivel del tercio medio del íleon (Figura 6). Se interpreta como cuadro de abdomen agudo obstructivo. Se realiza una laparotomía, y se identifica a nivel del íleon una formación nodular indurada blanquecina dependiente de la pared del intestino, que hace de pie de un vólvulo, por lo que se realiza una hemicolectomía derecha y anastomosis ileocólica.

Figura 6. Tomografía axial computarizada de abdomen con contraste oral y endovenoso. Se observan las asas del intestino dilatado con transición a nivel del ileon.

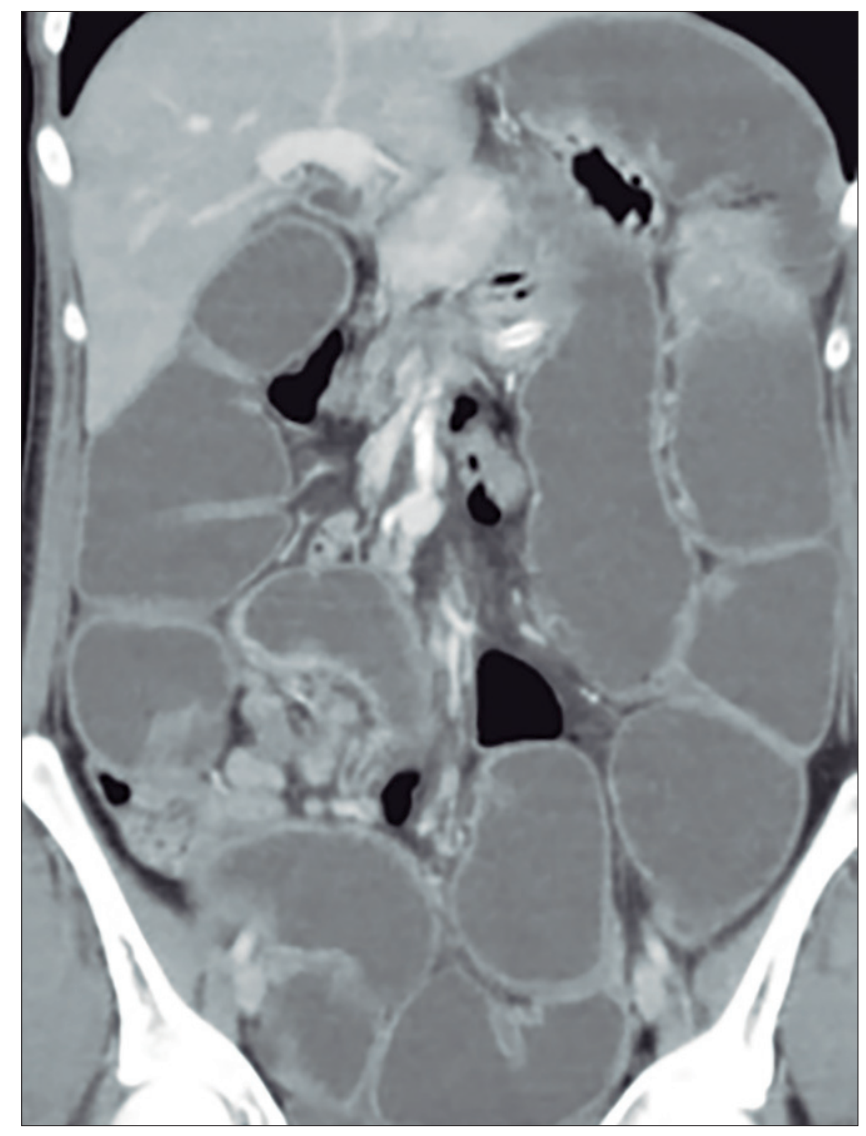

Evoluciona clínicamente estable, y se recibe el resultado de la anatomía patológica que informa endometriosis intestinal y apendicular. Ante el diagnóstico, se realiza una ecografía transvaginal en busca de otros focos endometriósicos, hallándose endometriosis focal, por lo que inicia tratamiento hormonal, con buena respuesta.

Actualmente se encuentra asintomática.

\section{Caso $\mathbf{N}^{\circ} 3$}

Paciente de sexo femenino de 40 años, sin antecedentes personales, que ingresa a la guardia por dolor abdominal localizado en fosa ilíaca derecha y vómitos de tres días de evolución. Niega otros síntomas asociados y no relaciona los síntomas con su ciclo menstrual habitual. Al examen físico de ingreso se evidencia un abdomen en tabla, con analítica de laboratorio con 10.056 glóbulos blancos $/ \mathrm{mm}^{3}$ y el resto sin particularidades. Se realiza una ecografía abdominal, que evidencia a nivel de la fosa ilíaca derecha, adyacente al ciego, un segmento intestinal aperistáltico, y una tomografía de abdomen que revela, en relación con el ciego, un segmento intestinal de paredes engrosadas de aspecto inespecífico.

Frente a un abdomen agudo secundario a sospecha de una lesión cecal, se decide realizar una laparoscopía exploradora, que evidencia peritonitis de cuatro cuadrantes, apéndice cecal y ciego engrosado con adherencias por implantes a nivel de íleon terminal. Se procede a realizar una hemicolectomía derecha, observándose pequeños implantes a nivel del intestino delgado, que son resecados y se biopsian.

Evoluciona clínicamente estable y días más tarde se observa en la anatomía patológica: apendicitis endometriosica con focos de endometriosis ileales con estenosis y adherencias interasas.

Con el propósito de evaluar otros focos endometriósicos, se realiza una ecografía transvaginal en la que se observan dos endometriomas de $2 \mathrm{~cm}$ en ovario derecho.

Inicia terapia hormonal con desogestrel, pero evoluciona con spotting permanente y proctorragia coincidente con los ciclos menstruales. Se realiza una resonancia de abdomen y pelvis con gel vaginal, con difusión y perfusión con contraste, observándose foco de endometriosis retrocervical, no visualizados por otros métodos (Figura 7).

Se rota el tratamiento hormonal a dienogest, con buena respuesta. La paciente persiste asintomática. 
Figura 7. Resonancia nuclear magnética de abdomen y pelvis con gel vaginal con perfusión y contraste, corte sagital. Foco retrocervical compatible con signos de endometriosis que contacta con el recto (flecha).

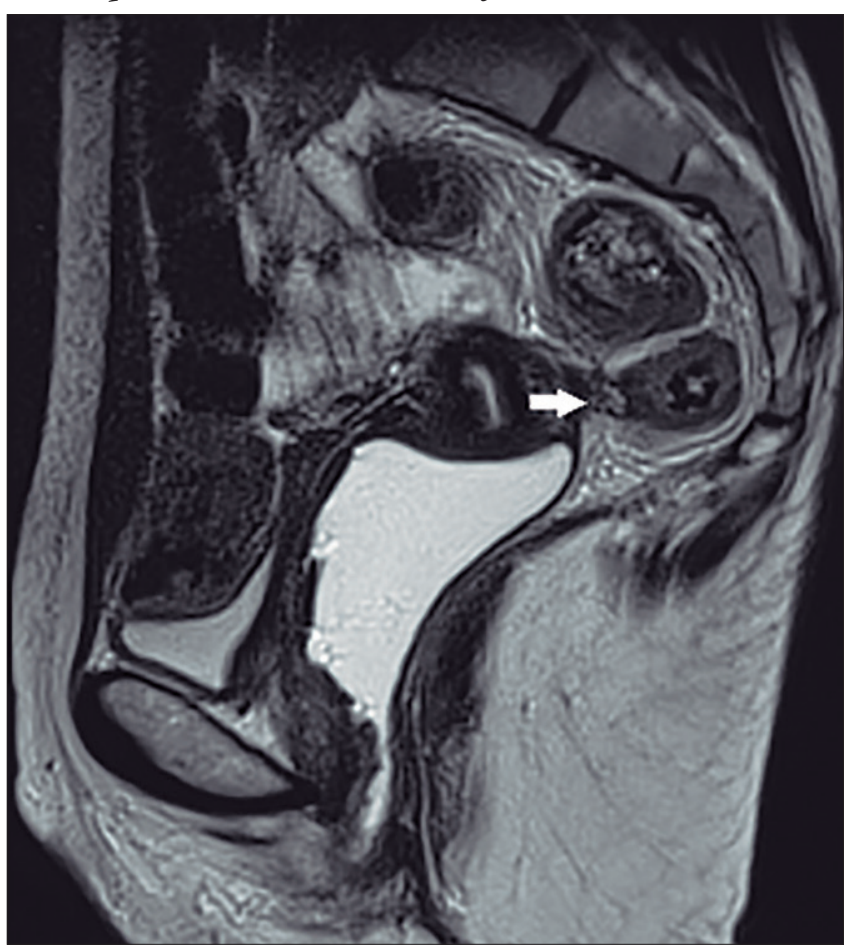

\section{Discusión}

Resulta de interés mencionar que la endometriosis puede clasificarse, en términos anatómicos, en genital y extragenital. La localización más común de la endometriosis extragenital es la intestinal y, en la gran mayoría de los casos, su presentación es asintomática. ${ }^{13}$

En el caso de las pacientes presentadas, coincidente con lo reportado en la literatura, se observa una ausencia de correlación entre los síntomas y el ciclo menstrual, que enmascara de esta manera el diagnóstico de EIPI y lleva a subdiagnosticar la entidad. ${ }^{7}$

La endometriosis como trastorno frecuente en las mujeres en edad fértil merece consideración a la hora de evaluar diagnósticos diferenciales de dolor abdominal, teniendo en cuenta que, en su presentación sintomática, puede mimetizar múltiples entidades de la gastroenterología, principalmente el SII o la enfermedad inflamatoria intestinal. Incluso con las herramientas diagnósticas avanzadas con las que contamos hoy en día, puede ser un gran desafío identificar la endometriosis intestinal. ${ }^{14}$

Sin embargo, sospecharla y poder diagnosticarla implica notorios cambios en la conducta terapéutica y la calidad de vida de nuestras pacientes. Si bien las terapias hormonales deben considerarse de primera línea, ya que son eficaces, seguras y bien toleradas, la escisión quirúrgica de la DIE debe tenerse en cuenta ante pacientes sintomáticas con fracaso del tratamiento hormonal. Siempre deben evaluarse los riesgos y beneficios que pueden surgir con posterioridad a la realización de una intervención quirúrgica. ${ }^{15}$

En los tres casos presentados, la oclusión intestinal por endometriosis se desarrolló en localizaciones anatómicas similares, afectando la región ileocecal, pese a no ser la localización más frecuente de compromiso intestinal, según lo reportado en la literatura. Más allá de no ser la localización más frecuente, es sabido que esta región anatómica posee angulación intestinal fija, lo que puede predisponer al desarrollo de cuadros oclusivos cuando se suma el compromiso endometriosico en dicha región, como en los casos de las pacientes presentadas.

Las tres pacientes tuvieron una buena evolución con el tratamiento quirúrgico instaurado y, posteriormente, la terapia hormonal en forma ambulatoria; es por esto que el abordaje multidisciplinario con el servicio de Ginecología y Cirugía General es de crucial importancia para la continuidad del cuidado de pacientes que debutan su enfermedad con requerimientos de cirugías en contexto de un cuadro de abdomen agudo.

\section{Puntos clave. Mensajes de aprendizaje}

- Es de relevancia conocer que la endometriosis intestinal es una de las localizaciones más frecuentes dentro de las manifestaciones extragenitales de esta enfermedad.

- Entender esta patología como una posibilidad diagnóstica a la hora de abordar los cuadros de dolor abdominal y/o suboclusivos en mujeres en edad fértil es fundamental para encarar opciones diagnósticas y terapéuticas poco frecuentes.

- La correlación entre los síntomas y la asociación con el ciclo menstrual no siempre es tan clara y es poco sensible como única estrategia para sospechar la enfermedad.

- Un diagnóstico temprano de esta entidad y su correspondiente tratamiento podría disminuir la necesidad de cirugías de urgencia en contextos desfavorables y la morbilidad que ello implica.

Conflictos de interés. Los investigadores de este trabajo declaran no presentar ningún tipo de conflicto de interés.

Soporte financiero. Los autores no recibieron soporte financiero alguno para la realización de este manuscrito. 
Consentimiento para la publicación. Para la confección de este manuscrito, se utilizaron datos anonimizados que no han distorsionado su significado científico.

Propiedad intelectual. Los autores declaran que los datos y las figuras presentes en el manuscrito son originales y fueron realizados en sus instituciones pertenecientes.

\section{Aviso de derechos de autor}

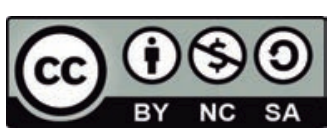

(C) 2021 Acta Gastroenterológica Latinoamericana. Este es un artículo de acceso abierto publicado bajo los términos de la Licencia Creative Commons Attribution (CC BY-NC-SA 4.0), la cual permite el uso, la distribución y la reproducción de forma no comercial, siempre que se cite al autor y la fuente original.

Cite este artículo como: Pérez T, Alsina Farreta $R L$, González ML y col. Endometriosis intestinal, una causa de suboclusión intestinal poco sospechada. Serie de casos. Acta Gastroenterol Latinoam. 2021;51(4):431-6. https://doi. org/10.52787/RJPF3089

\section{Referencias}

1. Bong JW, Yu CS, Lee JL, Kim CW, Yoon YS, Park IJ, Lim SB, Kim JC. Intestinal endometriosis: Diagnostic ambiguities and surgical outcomes. World J Clin Cases. 2019;7(4):441-51.

2. Nezhat C, Li A, Falik R, Copeland D, Razavi G, Shakib A, Mihailide C, Bamford H, DiFrancesco L, Tazuke S, Ghanouni P, Rivas H, Nezhat A, Nezhat C, Nezhat F. Bowel endometriosis: diagnosis and management. Am J Obstet Gynecol. 2018;218(6):549-62.

3. Soliman AM, Surrey E, Bonafede M, Nelson JK, Castelli-Haley J. Real-World Evaluation of Direct and Indirect Economic Burden Among Endometriosis Patients in the United States. Adv Ther. 2018;35:408-23.
4. Shafrir AL, Farland LV, Shah DK, Harris HR, Kvaskoff M, Zondervan K, et al. Risk for and consequences of endometriosis: A critical epidemiologic review. Best Pract Res Clin Obstet Gynaecol. 2018;51:1-15.

5. Ferrero S, Stabilini C, Barra F, Clarizia R, Roviglione G, Ceccaroni M. Bowel resection for intestinal endometriosis. Best Pract Res Clin Obstet Gynaecol. 2021;71:114-28.

6. Bray-Beraldo F, Pereira AMG, Gazzo C, Santos MP, Lopes RGC. Surgical Treatment of Intestinal Endometriosis: Outcomes of Three Different Techniques. Rev Bras Ginecol Obstet. 2018;40(7):390-6.

7. De Cicco C, Corona R, Schonman R, Mailova K, Ussia A, Koninckx P. Bowel resection for deep endometriosis: a systematic review. BJOG. 2011;118(3):285-91.

8. Ruffo G, Crippa S, Sartori A, Partelli S, Minelli L, Falconi M. Management of rectosigmoid obstruction due to severe bowel endometriosis. Updates Surg. 2014;66: 59-64.

9. Bazot M, Lafont C, Rouzier R, Roseau G, Thomassin Naggara I, Darai E. Diagnostic accuracy of physical examination, transvaginal sonography, rectal endoscopic sonography, and magnetic resonance imaging to diagnose deep infiltrating endometriosis. Fertil Steril. 2009;92:1825-33.

10. Yin S, Lin Q, Xu F, Xu J, Zhang Y. Diagnosis of Deep Infiltrating Endometriosis Using Transvaginal Ultrasonography. Front Med (Lausanne). 2020;7:567929

11. Bazot M, Kermarrec E, Bendifallah S, Daraï E. MRI of intestinal endometriosis. Best Pract Res Clin Obstet Gynaecol. 2021;71:51-63.

12. Bazot M, Detchev R, Cortez A, Amouyal P, Uzan S, Darai E. Transvaginal sonography and rectal endoscopic sonography for the assessment of pelvic endometriosis: a preliminary comparison. Hum Reprod. 2003;18:1686-92.

13. Camran Nezhat, Anjie Li Rebecca Falik, Daniel Copeland. Bowel endometriosis: diagnosis and management. American Journal of Obstetrics and Gynecology. 2018;:549-62.

14. Habib N, Centini G, Lazzeri L, et al. Bowel Endometriosis: Current Perspectives on Diagnosis and Treatment. Int J Womens Health. 2020;12:35-47.

15. Ferrero S, Alessandri F, Racca A, Leone Roberti Maggiore U. Treatment of pain associated with deep endometriosis: alternatives and evidence. Fertil Steril. 2015;104(4):771-92. 